\title{
Analysis of Possibility Theory for Reasoning under Uncertainty
}

\author{
Andrew A. Alola ${ }^{1}$, Mustafa Tunay ${ }^{1} \&$ Violet Alola $^{2}$ \\ ${ }^{1}$ Department of Applied Mathematics and Computer Science, Eastern Mediterranean University, North Cyprus \\ ${ }^{2}$ Department of Marketing Management, Eastern Mediterranean University, North Cyprus \\ Correspondence: Andrew A. Alola, o/c Mustafa Tunay of Faculty of Education, Eastern Mediterranean University, \\ North Cyprus. Tel: 90-533-886-6201. E-mail: andrewalola@ymail.com
}

\author{
Received: December 11, 2012 Accepted: January 31, 2013 Online Published: February 4, 2013 \\ doi:10.5539/ijsp.v2n2p12 URL: http://dx.doi.org/10.5539/ijsp.v2n2p12
}

\begin{abstract}
This work is purposed to analyze the idea of possibility theory for reasoning under uncertainty. This cut across the varying relationship and difference between probability and possibility theories as frequently studied. Various areas of application of possibility theory in modern fields as well as its use in modeling uncertainty were also painstakingly examined and alongside citing its application in forecasting productions in the energy sector as a useful illustration. Quantitative and Qualitative possibilities which are modeling techniques of the theory of possibility were as well studied and consciously differentiated with appropriate references to important parameters and supporting features like the possibility measure, possibility distribution and the imprecise probability.
\end{abstract}

Keywords: possibility, imprecision, probability, possibility measure, possibility distribution

\section{Introduction}

Although early in the 50's, economist G. L. S. Shackle had once proposed and worked on a non-probabilistic model of expectation, his works could not actually be seen as an alternative to probability theory-this type of uncertainty which is an alternative to probability theory is now widely and mathematically accepted to be a possibility theory. The principle and basis of possibility theory were thereafter conceptualized and put forward by professor Zadeh in 1978. Zadeh got his idea of possibility theory from his previous popular work on fuzzy sets and fuzzy logic which was earlier postulated in 1965. His previous works on fuzzy that addressed imprecision and variability of information with observers was later shown to generate analogues that hence relate the various properties of possibility theory and probability theory. The relation generated between these two theories which are based on set functions has been useful in the scope of reasoning statistically such as providing solution to information on uncertainty due to variability or vagueness and incomplete or imprecise information. Possibility theory is a modern and simple mathematical theory of uncertainty that is sufficiently used in the approach of incomplete information or imprecise probabilities. In possibility theory, a pair of dual set functions or subgroup of belief measures-that is necessity and possibility measures are used as alternative to a single set function in probability. It's a measure, indeed measures of non-specificity, because Hartley information which is relatively obscured or often ill-conceived is hugely and can be generalized only through broadening the notion of possibility theory (Klir \& Folger, 1992). Just as probability theory has a wider application to uncertainty problems, so is possibility theory to uncertainty due to imprecise information. A specific application of this theory is studied in this research.

\section{Review of Existing Literature on Possibility Theory and Its Applications}

In this chapter, several past works on possibility theory and related subjects are further reviewed so as to have an appropriate and broader view on the knowledge of possibility theory and its general applications. Apart from review the importance of possibility theory, its applications in different fields such as health and environmental sciences, information technology/engineering sciences, and economics/everyday life are made known.

In General, (Zadeh, 1978), the theory of possibility is related to the theory of fuzzy sets by simply illustrating the concept of a possibility distribution as a fuzzy restriction which acts as an elastic constraint on the values that may be assigned to a variable. De Cooman and Aeyels (2000) stated that is a study on the random set description of a possibility measure and its natural extension. The modeling of positive and negative information in possibility theory (Benferhat, Dubois, Kaci, \& Prade, 2008) is studied and observation is made precisely in a well defined way using the possibility theory. It is observed illustratively that a disparity can be acertained between what is 
supposedly possible as a result of inconsistency with the available knowledge and what is actually possible as often revealed from observations on the other hand. And the natures of information obtained are representatively coded using the necessity measures and possibility function which are the two types of constraints in this discourse. The research of Li and An (2009) suggests a new and successful method with a new idea into the knowledge of fuzzy sets and uncertainty is considered. This method, rough set model which is based on possibility measure is applied to overcome the problem and the inadequacies that have persisted long time before now resulting from the use of the ordinary and existing rough sets model especially in data reduction and in other analysis as regard possibility characteristics.

The use of this new method has made it easy to overcome these limitations and hence rough set is been nowadays used extensively in different fields of study to enhance effectiveness and accuracy in, for example, data mining and artificial intelligence among many. An interrelationship that differentiates possibility theory from probability theory is discussed by Dubois (2006) with a form of methodological reasoning in statistics especially in differentiating between uncertainties due to variability in information and incomplete information or observation. It is shown that certain situations in general statistics are illustrated using possibility theory language or simply encoded probability measures. An example of such probabilistic inequalities is the Chebychev's which is naturally encoded with poor or lack of probabilistic information that is used for formatting possibility distributions. Also, satisfying possibility theory are special types of likelihood functions especially which are characterized by unavailability of prior probability. Also Vejnarova (2002) stated that the theories of possibility and probability are linked with the use of Markov properties and factorization of possibility distribution which is use as an important implement in formulating multidimensional possibility distributions using a lower dimensional type. This idea of using a multidimensional possibilistic model is to format an effective analogies and as a solution to many possibility framework. And in achieving this, a specialized type of conditional possibility distributions is used to introduce the Markov properties and also in the factorization of possibility distributions which then generate a relation that shows an analogies result. In the work of Coletti and Vantaggi (2006) the notion of conditioning that is prominent in the study of probability is implored and used as one of the tools in discussing possibility theory in the context of conditional possibility.

\subsection{Health and Environment Sciences}

Prade and Dubois (1988) worked on the presentation of the inter-connectivity and dependency of belief functions and possibility measures as another interesting way of expressing a form of uncertainty. Because of the importance of the state of human health and his environment and the ever challenging situation of finding solution to many wellknown health risks diseases and environmental issues in the present day, researches has revealed the application of possibility theory to proffering significant solution to some of these issues. Also known about the application of possibility theory is in the framework of imprecision in health as researched by Buisson, Farreny, and Prade (1985) and even on life-risking illnesses. Imprecision knowledge which is highly in use in medical field has been a revelation to finding solution to the cure and management diabetic patients. The recent development of medical expert system, the DIABETO, is presently used for the treatment of diabetes and this is possible because of the imprecision and uncertainty of information of the medical knowledge. Kejiang (2010) studied the idea of mathematical modeling as implored and used in assessment of danger involved in contaminated sites especially in the framework of uncertainty and variability. The method of propagating hybrid of uncertainty and variability is shown to be very useful in the study of flowing of ground and contaminated water which can constitute a health issue to human life. This hybrid which is the transformation that takes place within parameters of uncertainties, i.e. probability and possibility variables are hugely analyzed to agree with the evidence theory and are observed to imitate the process of spread or rate of contamination of an underground water system. The concept of imprecise probability is shown and proved to be very important in the study of the environment ( $\mathrm{Fu}, \mathrm{Butler}, \mathrm{Khu}, \&$ Sun, 2011) especially in the flooding sewerage pattern of the environment.

\subsection{Information Technology and Engineering}

Obviously just as in the case of theory of probability and statistics where they are of significant importance in information and technology and also in engineering, it is now evident that the theory of possibility is also relevant in these fields. Umano and Fukami (1994) discussed the fuzzy data in the real world in the framework of fuzzy concept is used to formulate a possibility-distribution-fuzzy-relational model where the special type of relational algebra for the databases, Codd, is associated with the types of operations. Another important way where possibility theory and distribution are been useful for communication and networking purpose is in a retrieval system (Boughanem, Brini, \& Dubois, 2009). An information retrieval model is discussed in the framework of a directed 
network produced through possibility theory. This is built on a query system which identifies the relevance and use of the information by simply assigning the degree of necessity and possibility to carry out the evaluation differently. Rough set theory (Liu \& Zhong, 2001) is applied as a solution to imperfect data in inductive logic programming especially because the application of rough problem settings ease the necessary requirements in the standard normal problem setting for the inductive logic programming (ILP) to obtain more useful but rough information from such imperfect data. In several methods used for modeling uncertainty with much consideration on the analysis of some of the measures which are supposed to be relevant models for uncertainty in expert systems (Walley, 1996), the analysis is basically the comparison of the measures, i.e. possibility measures, coherent lower previsions, additive probabilities and belief function which thus revealed that only the lower and upper previsions types of measures are believed to be absolutely capable to model most known types of uncertainty. In their work Chung, Wang, $\mathrm{Xu}, \mathrm{Hu}$, and Lin (2007) the exponential possibility model which offers clustering concept through a set algorithm is discussed as also a method of data formulation. The use of this new clustering concept is very successful in artificial datasets and large image segmentation. Method of uncertainty is also known to be useful in the analysis of prognostics (Baraldi, Popescu, \& Zio, 2010).

Analysis of prognosis especially on system, structure or component is carried out mostly to forecast its effectiveness and otherwise its loss of functionality by simply studying the time of failure. A Hybrid Monte Carlo and possibilistic method alternatively carried out the representation and propagation of the type of uncertainties involved with such forecast. Both Zadeh's possibility theory and fuzzy sets are extended to be used in prognostic analysis of fault in satellites (Cayrac, Dubois, \& Prade, 1996). Extent and effect of the fault diagnosis activities are normally investigated using the Fault Mode Effects and Criticality Analyses (FMECA). In Kim's (2009) study, one of the useful applications of uncertainty in engineering is shown in the serviceability of reinforced concrete structures (RCS). Evidently, reinforced concrete properties, i.e. concrete materials and mixing and casting method among many others are widely known to have a varying inconsistencies and uncertainties and such properties are modeled as random uncertainties using the theory of probability.

\subsection{Economics and Real Life Problem}

The importance of possibility theory especially using the perception of probability and statistics intuitive especially in the economic sense cannot be overlooked. Theory of possibility like probability is applied in the study and analysis of risk, ranking alternatives, decision making and even in investment among others interesting applications. Investment options abound in our everyday activities, but in Mohamed and McCowan (2011) study, forecasting or making a suitable decision as a way of overcoming the challenges posed by such options requires modeling of such investment decision under uncertainty through the application of possibility theory. A procedural evaluation of investment options is critically important in any capital resources based project so as to arrive at or make use of the most appropriate option for such investment. And it is simply proposed that the uncertainties encountered in this type of challenge are represented using possibility theory through modeling of the effects of both monetary and non-monetary attributes of options of such investment. In Mukerji (2000) study, the application of the concept of ambiguity aversion in economics is studied. Just like a decision maker that is not risk loving is always prompted to averse risk especially when confronted with a risk situation, so is ambiguity aversion idea is applied especially when there is an imprecise or incomplete information of an event or in a subjective uncertainty. Because of this imprecise information, the ambiguity averse decision maker exercises caution in making his decision and such motive is applicable in several economic-related situations like strategic decision making in auctions and in the design of bilateral economic contracts just to mention few.

Also, in Gerard, Kaci, and Prade (2007) the approach of possibility theory is expressed and analyzed as a method of ranking of ordered alternatives that are strictly based on the specialized generic constraints. Relatively, these ranking of the alternatives are ordered with respect to the comparison of their individual states without imploring the method of an aggregation operation in the evaluation of alternatives such as in a multi-criteria decision making (MCDM) process. Rebille (2006) also tailored the concept of Choquet integral as used in decision making over a necessity measure by simply ranking measures with the use of its Choquet expected utilities. This method is relatively similar to Von Neumann and Morgenstern's method of decision making under risk, and provides a category of information which is made available by its Choquet expectation of the fuzzy set.

\section{Uncertainty in Possibility and Probability Theory}

\subsection{Data Uncertainty and Imprecision}

It is necessary to discuss the nature of unique relationships that exist between probability and the fuzzy set type of 
possibility theory. Traditionally, data uncertainty and imprecision of information are always modeled effectively with the use of classical probability and hence, normally imprecise data is often presently linked or used as its modeling tool. Based on this form, probability is always regarded as the likelihood (frequency of occurrence) of an occurrence with respect to an alternative outcome known as the most frequentist representation (Winkler, 1972). This type of definition of data uncertainty and imprecision is attributed with the general knowledge of system randomness. But over the year comes the development of alternatives means of data imprecision representation that includes Bayesian interpretation of probability (better put as possibility theory), theory of evidence by DempsterShafer, and fuzzy set theory by Zadeh. The Zadeh's proposition-Fuzzy set theory is hence in contrast used to describe imprecision especially when there is incomplete information, or better say when there is data ambiguity as a result of subjective or linguistic judgments. In the further development of fuzzy set in this context comes the knowledge of possibility theory which is best regarded as the ideal representation of fuzziness which is given to a possibility value in contrast to probability value to show the extent that a particular outcome is indeed believe to happen. Hence, the degree of belief of event occurrence is always assigned a value from the interval $[0,1]$ in interpreting possibility theory.

\subsection{Analysis of Sources of Data Imprecision}

Since the whole idea behind this study is from the fact that it is absolutely inevitable to have a real life devoid of uncertainties, it is necessary the sources of these inaccuracies are discussed. Really, the source of incomplete data might be inexhaustible but we can streamline the causes of generating this imprecision to include: imprecision due to linguistics error (statements), data conflicts, incomplete knowledge and selection from data alternatives.

\subsubsection{Incomplete Knowledge or Data}

On many occasion, imprecise data can be as a result of lack of knowledge about a situation especially when there are unknown and contingent factors. Some of these unknown factors causing these uncertainties in imprecision decision modeling might be nature in-built (not practically measurable) and hence remained unresolved. For instance, when trying to make decision to influence the growth or possible change in the economy of a particular country over a long period of years by modeling using a specific uncertainty model, these type of modeling will be influenced certainly by certain unpredictable future factors like birth rate, global economy among many. Hence these unpredictable future factors are responsible for the incomplete information, and influence the result of the modeling.

\subsubsection{Selection from Data Alternatives}

In real life it is very common we are often engulfed with making decision and hence spend more precious time in arriving at concrete conclusion. This decision making problem is experienced in modeling uncertainty especially when it is necessary that selection has to be made among several alternatives of data or information. If a wrong data is selected among this available information, then the outcome of the modeled imprecision is affected.

\subsubsection{Data Conflicts}

This type of source of uncertainty is similar with the previously discussed type but differs in that accurate information might be available in a vague or unclear manner. The fact or information available might be conceivable from different or conflicting angle or meaning depending on the perception hence causes uncertainty in information. This can be illustrated by saying "economy is good" which really is an ambiguous statement in real context. Suppose the state of an economy is measured with its GDP (gross domestic product) using the interval $[0,1]$, then to really adjourn a particular economy to be good or bad, the GDP must indeed take value from above interval. Since more than one of these values can be assigned and will still implies a good economy especially when a value of GDP is higher or equal to the value of the interval, then there exists a conflicting information.

\subsubsection{Imprecision Due to Linguistic Statements}

It is a common way of human life to pass comments and unconsciously express opinion which might be interpreted or assigned different meaning by the listeners. When a speaker makes use of some imprecise terms (terms like might or should) to express their individual subjective knowledge, it is easily interpreted by unfamiliar listener to mean something different from the original context of the speaker and an uncertain information is hence passed across in that course.

This source of imprecision is responsible for 'Linguistic' uncertainty as against 'Epistemic' type which is caused by error, variation or imperfection of data measurement. 


\subsection{Axiomatic and Comparative Analysis of Probability and Possibility Theories}

Although, some aspect of probability like the Bayesian probability are closely related with possibility theory but also largely differs in major aspect especially in its axiomatic foundations. This axiomatic difference is observed if a fuzzy measure is generally used in place of real measures such that on a finite universal set, probability and possibility are expressed by fuzzy measures (Klir \& Yuan, 1995). Fuzzy measure is a continuous or semi-continuous function taken from a class of crisp sets of a given power set associated with the interval [0,1], (Nikolaidis, Chen, Cudney, Haftka, \& Rosca, 2004). Importantly, the axiomatic properties of probability and possibility measures from the generalized fuzzy measure are centered about the boundary condition, the positivity or non-negativity and the additive nature as carefully expressed in the subsequent table.

Table 1. Additivity, non-negativity (monotonicity) and boundary condition properties of probability and possibility measures

\begin{tabular}{llll}
\hline & Additive property & Non-negativity property & Boundary condition \\
\hline Probability Measure $P(x)$ & $\sum_{i=1}^{n} P\left(A_{i}\right)=P\left(\bigcup_{i}^{n} A_{i}\right)$ & $P(A) \geq 0$ & $P(U)=1$ \\
Possibility Measure $\prod(x)$ & Maxi $\in$ G & Given $A, B \in U$, if $A \subseteq B$, & $\prod(\phi)=0$ and \\
& $\left\{\prod\left(A_{i}\right)\right\}=\prod\left(\bigcup_{i}^{n} A_{i}\right)$ & then $\prod(A) \leq \prod(B)$ & $\prod(U)=1$ \\
\hline
\end{tabular}

The Table 1 shows the additivity, monotonicity (non-negativity) and boundary condition properties of probability and possibility measures ( $A$ and $B$ are events, and $A_{i}$ represent the partition of a universe of discourse $U$ ). For instance, in principle the union of the probabilities of more than one disjoint event (unlimited number of events) is equal to the probability of the union of the events and fundamentally summed to one (additive property). This absolutely contradicts the theory of possibility in that there is no additive relation between events and hence the possibility of a finite union of events (or outcomes) are independent and equal to the maximum of the possibilities of these events (sub-additive or the Maxitive and Minitive property) and also not necessarily equal to one. Instead, the possibility of the events of the universe of discourse $U$ is always greater or equal one, i.e. $\sum_{i}^{n} \Pi\left(A_{i}\right) \geq 1$.

Both uncertainty theory types above are based on set-functions but a pair of dual set functions named possibility and necessity measures are associated with possibility theory as against one set function in probability. From the illustration of Maxitive and Minitive of possibility theory above, if the possibility degree of 'disjointed' certain occurrences is given as maximal value of degree of possibility of the disjuncted occurrences, then the degree of necessity of 'conjoined' occurrences would be the minimal value of the degree of necessity of such occurrences of the conjunction.

\subsection{Theoretical Comparison in Modeling Methods of Uncertainties}

It is really necessary to elaborate how the forms of the uncertainties presented are modeled. If the source of imprecision is well-established, the best and effective way it is represented can be easily outlined. The idea of modeling of uncertainty which led to the development and more research on possibility theory is very important because of the continuous disparity in the presentations of the principles of probability as perceived by both objectivist and subjectivist especially when dealing with uncertainty. While both classes of thought reasoned that probability measure is the only reliable mathematic tool for modeling uncertainties, yet the ideas put forward as regards the modeling pattern by these different classes (objectivist and subjectivist) differs. While the objectivist argued for modeling of unknown uncertainties that are completely deterministic like saying "the actual maximum height an unborn baby will attain in life", the subjectivist identifies that probabilities are tailored to model those uncertainties that have a form of complex or ambiguous variability like saying "women weight in a certain country". The former example of the objectivist above, "the actual maximum height an unborn baby will attain in life", is also a sure uncertainty issue as a result of lack or insufficiency of knowledge of actual maximum height the unborn baby will attain, because is an occurrence that pertains to the future.

The later example of the subjectivist above, "the weight of women in a country", is obviously an imprecision caused by variability in the information on women's weight in the country because different women will largely have different weight values. There seems to be disparities in modeling pattern or method of uncertainty based on variability of information and lack of information. Nevertheless, it is unclear that lack of information could be modeled with the use of similar tools when complex variability of information by the objectivist and subjectivist is respectively in consideration as presented by Hoffman and Hammonds (1994) and Dubois, Prade, and Smets (1996). Because of the concern that a classical probabilistic tool might not be enough to model such uncertainty resulting from incomplete or lack of knowledge as compared with modeling of variability of information, then pos- 
sibility theory is currently devoted for such modeling because of its mathematical simplicity. Possibility theory is then seen as a rough non-numerical version of probability or best expressed as a simple methodology to reasoning with imprecise probabilities (Dubois \& Prade, 1992). Other effective and classical methods of handling uncertainties include the use of certainty factor (CF) using degree of belief and Dempster-Shafer theory of evidence using belief and plausibility measures.

\section{Method}

\subsection{Handling Uncertainties Using Probability and Possibility Theories}

Over few centuries from now probability theory has been thoroughly discussed and some general thoughts about it have been expressed mathematically, in the view of frequentist and the Bayesian knowledge. In general, apart from the three major axiomatic fundamentals of both the probability and possibility theory as discussed earlier, some other mathematical tools are also considered to be very effective in handling uncertainties using classical probability theory. This method could include joint probability, conditional probability with Bayes' rule, conditional independence and probability density function. One of these - the conditional probability and with Bayes' rule is illustrated as follows:

What is the probability that the car will break down given that it is old?

Let $A$ represent event that the car will break down. Let $B$ represents event that the car is old. So that if $P(A / B)$ is the probability of happening of $A$ where $B$ has already happened. $P(A \cap B)$ is the probability of happening of $A$ and $B$ at same time. $P(B)$ is the probability of happening of $B$ alone. Then relation to express the conditional probability of the statement above is

$$
P(A / B)=\frac{P(A \bigcap B)}{P(B)}
$$

From the above expression, Bayes' rule can equally be manipulated mathematically and expressed for the probability "that the car will break down given that it is old" as:

$$
P(A / B)=\frac{P(B / A) \cdot P(A)}{P(B)}
$$

The essence of two approaches to this theory is to propose a model or reasoning for uncertainties caused by imprecise or lack of knowledge. The first one, Zadeh's approach is intuitively plausible and from the perspective of fuzzy set theory which associates a level of fuzziness to possibility theory. Also, the second approach to handling uncertainties using possibility theory is the axiomatic approach which is just an extension and further development of the fuzzy set approach. The main idea behind the fuzzy set approach is the relation between the membership function $\mu_{f}(U)$ of the fuzzy set $F$ in a universe of discourse $U$ and the possibility distribution $\pi_{x}$ and hence the relation denotes that $\pi_{x}$ is numerically defined to equal $\mu_{f}(U)$, i.e. $\pi_{x}=\mu_{f}(U)$. In expressing the axiomatic approach, the tools of Dempster-Shafer body of evidence which are the belief (bel) and plausibility (pl) measures are used for modeling the uncertainty. Simply, the belief and plausibility measure are changed to the pair of dual set of functions which are the necessity measure $(\eta)$ and the possibility measure $\left(\prod\right)$, respectively.

Given two possible occurrences $A$ and $B$ with degree of uncertainties, the dual set functions of measures are interpreted as follows: $\operatorname{Bel}(A \cap B)=\operatorname{Min}[\operatorname{bel}(A), \operatorname{bel}(B)]$ is represented as $\eta(A \cap B)=\operatorname{Min}[\eta(A), \eta(B)]$ and $\operatorname{Pl}(A \cup B)=\operatorname{Max}[\operatorname{pl}(A), \operatorname{pl}(B)]$ is represented as $\prod(A \cup B)=\operatorname{Max}[\pi(A), \pi(B)]$. Also from its axiomatic properties comes another relation which shows the property of duality relationship between necessity and possibility measures. Then for the same event $A \in U, \eta(A)=1-\prod\left(A^{c}\right)$ where $A^{c}$ represents the complement of the event $A$.

\subsection{Imprecise Probability}

Far from the usual classical or rather a precise representation of uncertainties which can be regarded as an extension to a precise form of probability is known as the imprecise probability. In the nineties, the foundation of imprecise probability was coined by Peter Walley from the previous works of Kuznetsov and Weichselberg when the similar form of uncertainty was referred as an interval probability. Here point values are not assigned to event to form its probability distribution as noted in Kolmogorov's axioms but rather it expresses a generalization of the possibility theory with modifications and simply applicable when there is scarcity or conflict in available information.

It is widely acknowledged that the quality or exactness of information can hardly be represented accurately with the use of a single probability measure since a form of "cloud" as proposed by Neumaier is believed to exist within such interval probabilities. Such representation with two set-functions-likes is named as the lower and 
upper probabilities. For instance, if the classical probability of $\mathrm{B}$ happening is given to be $\mathrm{P}(\mathrm{B})$, then its upper and lower probabilities are $\bar{P}(B)$ and $P(B)$, respectively. Apart from the unique situation when the lower and upper probabilities are equal signifying a precise probability, i.e. $\bar{P}(B)=\underline{P}(B)$, otherwise $\bar{P}(B)<\underline{P}(B)$. The upper and lower probabilities are defined on the interval $[0,1]$. In the presence of complete lack of knowledge for the same event $B$ the $\bar{P}(B)$ and $\underline{P}(B)$ get upper and lower bounds of the probability, i.e. 1 and 0 , respectively (Coolen, Augustin, \& Troffaes, 2010). The new generalization of precise probability to extend the modeling approach of uncertainty using probabilistic-based model has wider applications ranging from statistical inference, nonparametric predictive inference to logical probability and critical decision analysis and also its limitations still poses major challenges as well.

\subsection{Comparative Advantage of Modeling Uncertainty with Possibility Theory}

Since this research work is based mainly on possibility theory, it is quite necessary to briefly discuss the main advantages of using possibility approach over probability in handling uncertainty. A part from the known fact which shows the non-dependency of the possibility of an event on an alternative event and hence makes possibility approach of handling uncertainty to be less restrictive, there exists few main justifications of the theory of possibility over the usual mathematical modeling of probabilities (Raymond, Alvin, \& Michael, 2002). The subjective and heuristic data inputs nature of the possibility theory makes it more intrinsically compatible. This is true because small data inputs and the use of the concepts of fuzziness like the subjectivity, flexibility and plausibility makes it easy to generate possibility distributions. Although the simplicity and mathematically less restrictive nature of the use of possibility theory might be disadvantageous in some context, but this features make it more significantly less rigorous and economical in computation when used to propagate uncertainty in expert system. An example is the difficulty and the rigorous analytic nature of propagating uncertainty in life-cycle inventory analysis using probabilistic method-based approach (Maurice, Frischknecht, Coelho-Schwirtz \& Hungerbuhler, 2000). Lastly, if probability is referred to as the frequency of occurrence of an event which makes such outcome or observation as ordinary outcome of a random process, then the approach is less accurate and less effective when especially imprecision results from ambiguity as oppose to randomness. Because the validity of a modeled system hugely depends on the accuracy of the fundamental principles (Borenstein, 1998), then the most appropriate approach to imprecision is used. The possibility theory approach is obviously and effectively considered to be more accurate in handling uncertainty of data imprecision that is void of randomness.

\subsection{Analytic Classification of Possibility Theory}

This special trend in uncertainty as opposed to probability theory captured the modeling of uncertainty as the epistemic of partial belief and classified semantics to certainty and plausibility. Plausibility is known to have a dual relation with certainty (Gabbay \& Smets, 1998) because while the former is termed 'lack of surprise', the latter clearly reflects a lack of plausibility of an event. The specifics of the varying properties of this form of uncertainty representation made way for the painstakingly analyses and further classification of possibility theory. Hence, the respective objective and subjective classification of the specifics of possibility theory as quantitative and qualitative further emphasizes the strength of the theory as based on the principle and the use of dual set-functions as opposed to a set-function of the theory of probability. Although these forms of possibility theory are highly compatible when modeling with the set-functions but a thin and definite line exists in both their conditioning and combination tools as well as the detail analysis of their main properties.

\subsubsection{Quantitative Possibility}

The objective form of possibility theory is related and categorized as a special resemblance of a generalized type of probability theory with inadequate statistical information and a unique representation of the degree of belief of both the possibility and necessity because these degree values are from the unit interval [0,1]. Quantitative possibility theory is seen to be able to model quantified imprecision as evident in the representation of the degree of uncertainty of possibility theory based on both the subjective (belief generated from an individual judgment) and objective (belief generated from data or statistical observation) views. Although, both context proposes to represent degrees of belief but the presence of imprecise statistical inferences makes quantitative possibility devisable for the approximation of lower and upper frequentist probability (probability distribution) using the objective context (Kruse \& Gebhardt, 1993). Quantitative possibility theory is comparatively represented like the Bayesian of a probabilistic model and as transferable belief model both within the subjective context (Smets \& Kennes, 1994). In analyzing dual set-functions among many methods, a relation is established using idempotent analysis and also with the use of possibility measure which is related to probability theory that has large deviations values. 


\subsubsection{Qualitative Possibility}

This form of uncertainty analysis which is more of a non-monotonic reasoning is simply a direct method of avoiding challenges resulting from errors in measurement. Comparatively, the set-functions on an ordered scale $L$ or partially ordered states of events of a finite state of space are represented with the use of a linguistic interpretation known as the qualitative possibility theory. Similar to the ordering formation of possibility distributions, here the plausibility ordering represents a total partial or pre-order with the use of $\geq_{\pi}$ that induces a well-ordered partition $\left\{E_{1}, E_{2}, \ldots, E_{n}\right\}$ of finite universe set $S$ that is expressed analogously as $s \geq_{\pi} s^{1}$ if and only if $\pi(s) \geq_{\pi} \pi\left(s^{1}\right)$ (Dubois \& Prade, 2011). The respective notations and relations of possibility and necessity measures are stated as $\Pi$ and $N$ such that the possibility measure $\prod$ which uniquely depends on possibility distribution $\pi$ and related as $\prod(A)=\max _{s \in A} \pi(s)$. Illustrating a challenge or linguistic problem qualitatively, we assume a question "has the situation $A$ occurred?", where $A$ is a member of the universe $S$ of an ordered scale $L=[0,1]$. Then $\prod(S)=\sup _{s \in A} \pi(s)$, and $N(A)=i n f_{s \notin A} 1-\pi(s)$. Since possibility measures expresses the degree or extent of consistency and necessity categorize implication of an event, then $\Pi(A)$ gives the extent to which $A$ is consistent and $N(A)$ denotes how certainly $A$ is implied with the distribution $\pi$. Recall that the dual set property is illustrated as $N(A)=\prod\left(A^{c}\right)$, and also if $\prod(A \cup B)=\max (\Pi(A), \Pi(B))$ in comparison with $N(A \cap B)=\min (N(A), N(B))$ of possibility and necessity respectively, then qualitatively this can also be represented in the ordinal forms similar to the aforementioned. Hence $A \geq_{\Pi} B$ if and only if $\max (A) \geq_{\Pi} \max (B)$ and $A \geq_{N} B$ if and only if $\max \left(B^{c}\right) \geq_{\Pi} \max \left(A^{c}\right)$ are known to satisfy Lewis characteristic property [40].

\subsection{Possibility Measure and Distribution}

Possibility measures the degree by which a presented evidence of event occurrence does not contradict the fact or hypothesis that the event will eventually happen. An event that is sure to occur (can occur) is given a possibility of one and if otherwise a value zero is assigned. Then, proposition exists that the possibility of an event is the smallest value within given interval $[0,1]$ simply because possibility is considered as the upper bound of probability (Giles, 1982). If an imprecise statement like "Andrew's height is above $180 \mathrm{~cm}$ " is to be considered, then it is simply inferred that any height $h$ above $180 \mathrm{~cm}$ is possible for Andrew and any height $h$ less or equal 180 is absolutely impossible for him where possible takes the value one and impossible takes the value zero. This is represented by possibility measure defined on Andrew's height domain as illustrated below:

$$
\prod_{h}\left\{(\text { Andrew's height }(\mathrm{cm})\}=\left\{\begin{array}{l}
0, h \leq 180 \\
1, h>180
\end{array}\right.\right.
$$

Also, for any event there exists proposed condition that the possibility of an event should be greater or equal to its probability (Zimmermann, 1996). Obviously this is justified since any event that is probable must likewise be possible but the converse is not applicable.

The possibility distribution examines the degree of possibility of each possible value of an examined variable $X$ of an event which is single-valued, meaning that the elements of the origin of the variable $X$ are rank-ordered according to their relative possibility or level of degree on the scale $[0,1]$. When the values of these functions (possibility distribution functions) are restricted to $0 \mathrm{~s}$ and $1 \mathrm{~s}$, the measure is referred to a crisp possibility or necessity measures [1]. For example: If $X$ is a set such that there exists subset of $X$, then a bigger set consisting of all subsets of the given set $X$ which is called power set of $X$ will be $\rho(X)$. Also if every $x$ is a member of $X$ with a possibility $P o s$ on $\rho(X)$, then the possibility distribution function will be: $r: X \rightarrow[0,1]$. Given a fuzzy subset $A$ of $X$ with membership grade function $\mu_{A}$, Zadeh defines a possibility distribution $r$ associated with $A$ as numerically equal to $\mu_{A}$, that is $r(x)=\mu_{A}(x)$. The possibility distribution $\pi$ of the function $r: X \rightarrow[0,1]$ of the above statement is expressed as $\pi(A)=S u p_{x \in A} r(x)$ and $X$ is infinite for $A \in \rho(X)$. When $X$ is finite, the expression becomes $\pi(A)=\operatorname{Max}_{x \in A} r(x)$ which is then the fuzzy measure.

Consider the $X$ number of goals conceded by a certain football club say Chelsea FC in a single match of 90 minutes full-time. Let's assume that $X$ can take the values from the interval $U=\{0,1,2,3,4, \ldots, 7\}$. Then both the degree of belief, $\pi(u)$ with which Chelsea FC concedes $X$ number of goals, and $P(u)$ which represents the probability that indeed Chelsea FC will concede $X$ number of goals is illustrated in Table 2.

Table 2. Association of possibility $\pi(u)$ and probability $P(u)$ distributions with $X$

\begin{tabular}{lllllllll}
\hline$U \Rightarrow$ & 0 & 1 & 2 & 3 & 4 & 5 & 6 & 7 \\
\hline$\pi(u) \Rightarrow$ & 1 & 1 & 1 & 1 & 1 & 0.6 & 0.4 & 0.1 \\
$P(u) \Rightarrow$ & 0.3 & 0.3 & 0.2 & 0.1 & 0.1 & 0 & 0 & 0 \\
\hline
\end{tabular}


Obviously the values in the table above illustrate that high value of possibility does not indicate a corresponding high probability value but rather shows that a probable event is indeed possible and also that an impossible event is indeed impossible (not probable). The possibility and probability distributions are graphically represented in Figures 1 and 2, respectively.

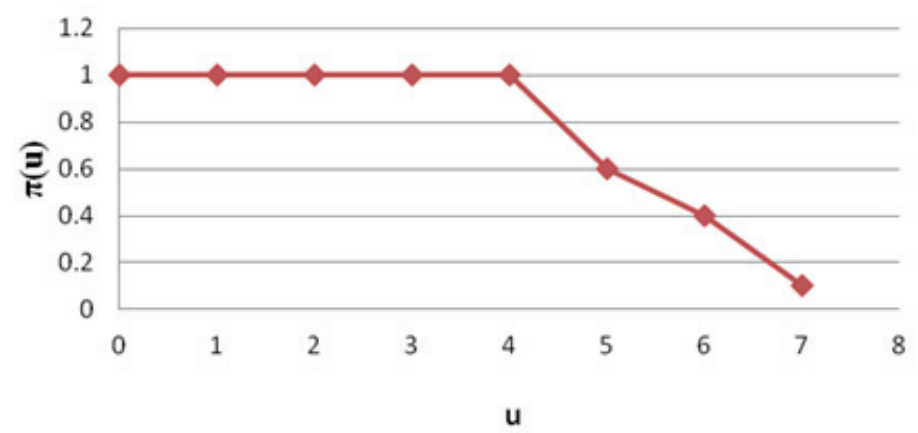

Figure 1. Possibility distribution

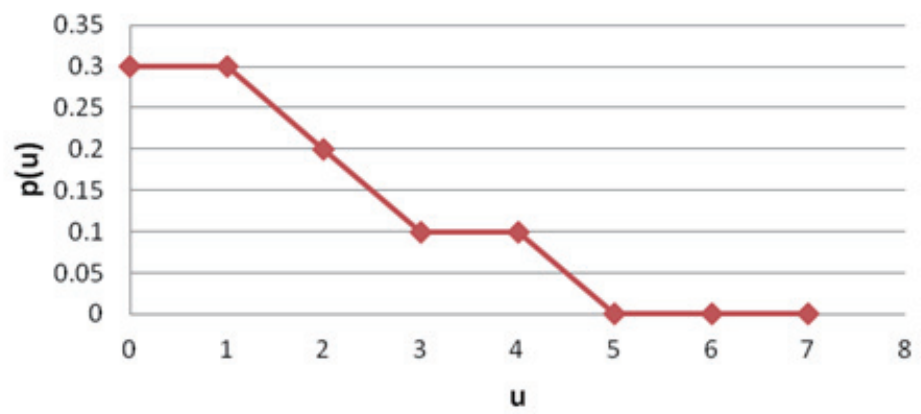

Figure 2. Probability distribution

Mainly, the relevance of analyzing uncertainty with possibility theory is better appreciated when evidence about events are quite unreliable or when prediction or conclusion is difficult to make due to insufficient information. The global energy demand projections as projected in Shell Energy Scenarios to 2050 and Chevron energy projection to 2035 among many other projections by different energy companies is illustrated in Table 3 showing the degrees of possibilities of demanding Crude oil, Natural gas, Coal and Nuclear by the year 2030 [43, 44, 45].

Table 3. Possibility distributions of global energy demands by the year 2030

\begin{tabular}{ccccc}
\hline & Crude Oil & Natural Gas & Coal & Nuclear \\
\hline$\pi($ Shell $)$ & 0.9 & 0.7 & 0.8 & 0.3 \\
$\pi($ Chevron $)$ & 0.4 & 0.5 & 0.9 & 0.2 \\
\hline
\end{tabular}

Let's try to express the possibility and necessity degree using only the distribution for Shell. Now if the universe of discourse associated with demand $\mathrm{d}$ is represented as $U_{d}$ and where $U_{d}=$ \{Crude Oil, Natural gas, Coal, Nuclear , and also $\mathrm{A}$ is a subset of $U_{d}$ which is given as $A=$ \{Crude Oil, Natural gas\}, then the possibility degree $\prod(A)$ of global energy demand d by 2030 where $d \in A$ can be expressed using the earlier expression. Hence from $\Pi(A)=\sup _{d \in A} \pi(d)$, the degree of possibility of global energy demand by the year 2030 is expressed thus: $\prod(A)=$ $\sup \{0.9,0.7\}=0.9$. Likewise the necessity degree is determined using the earlier expression such that $N(A)=$ $\inf \{1-0.8,1-0.3\}=\inf \{0.2,0.7\}=0.2$.

\section{Discussion}

Practically from the charts (Figures 1 and 2) which depicts the curves of possibility and probability distribution respectively, the resemblance in the nature of the curves relatively proof the importance and capability of possibility modeling as compared to the usual and classical method of probabilistic modeling of uncertainty. 
Just as mentioned earlier, modelings with possibility methods of a degree of uncertainty like that of the Global Energy demand of the year 2030 illustrated above the futuristic production and relevance of energy supply is hugely uncertain or variably unpredictable for such period of year and above. The value 0.9 for the degree of possibility above simply represents the extent of consistency in global energy demand by the year 2030 and likewise the value 0.2 illustrates the certainty of the same situation using only the projections provided by Shell Company. The same process can be implored to express the possibility and necessity degrees by different companies or data from different sources as well as used in the computation of huge projections about other energy types and even in many fields to proffer solutions to real-life problems.

\section{Conclusion and Suggestions}

In this paper, relevance of the theory of possibility for reasoning under uncertainty is comparatively analyzed with probability theory. It categorically shows the importance of the possibility theory in different modern fields and it importance especially to forecasting an uncertain situation given relatively an insufficient data as illustrated with the situation of the Chelsea Football Club of England and the Global Energy demand of 2030 as illustrated in this study.

The applicability of the theory of possibility in different fields especially in the modern world is huge. In real life where human future state of life (like the standard of living for a period of time of a nation) depends on so many factors like the fiscal policy of government, production cost and market values among many almost leading to an uncertain future, modeling with possibility theory of such uncertainties could help ascertain such future state. The advantages of the possibility theory over the probability theory in the presence of uncertain and imprecise nature of information are discussed. More research work as regard the classification of possibility theory into quantitative and qualitative forms is expected to be further considered with a recommendation that an extended study should focus on their individual application even beyond the scope of possibility theory.

\section{Acknowledgments}

All appreciation to the Editor, contributors, anonymous referees and indeed every member of my immediate family not leaving out-Victor Olatunji Alola.

\section{References}

Baraldi, P., Popescu, I. C., \& Zio, E. (2010). Method of uncertainty analysis in prognostics. International Journal of Performance Engineering, 6(4), 305-326.

Benferhat, S., Dubois, D., Kaci, S., \& Prade, H. (2008). Modeling positive and negative information in possibility theory. International Journal of Intelligent Systems, 23(10), 1094-1118. http://dx.doi.org/10.1002/int.v23:10

Borenstein, D. (1998). Towards a practical method to validate decision support systems. Decision Support Systems Publication, 23(3), 227-239. http://dx.doi.org/10.1016/S0167-9236(98)00046-3

Boughanem, M., Brini, A., \& Dubois, D. (2009). Possibilistic networks for information retrieval. International Journal of Approximate Reasoning, 50(7), 957-968. http://dx.doi.org/10.1016/j.ijar.2008.10.005

Buisson, J.-C., Farreny, H., \& Prade, H. (1985). The development of a medical expert system and the treatment of imprecision in the framework of possibility theory. Information Sciences, 37(1-3), 211-226. http://dx.doi.org/10.1016/0020-0255(85)90014-3

Cayrac, D., Dubois, D., \& Prade, H. (1996). Handling uncertainty with possibility theory and fuzzy sets in a satellite fault diagnosis application. Fuzzy Systems, IEEE Transactions on Fuzzy Systems, 4(3), 251-269. http://dx.doi.org/10.1.1.45.7378

Chung, F.-L., Wang, S., Xu, M., Hu, D., \& Lin, Q. (2007). Possibility theoretic clustering and its preliminary applications to large image segmentation. Soft Computing-A Fusion of Foundations, Methodologies and Applications, 11(7), 103-113. http://dx.doi.org/10.1007/s00500-006-0056-8

Coletti, G., \& Vantaggi, B. (2006). Possibility theory: Conditional Independence. Journal of Fuzzy Sets and Systems, 157(11), 1491-1513. http://dx.doi.org/10.1016/j.fss.2006.01.003

Coolen, F. P. A., Augustin, T., \& Troffaes, M. C. M. (2010). Imprecise probability. International Encyclopedia of Statistical Science. Springer.

De Cooman, G., \& Aeyels, D. (2000). A random set description of a possibility measure and its natural extension. 
Systems, Man and Cybernetics, Part A: Systems and Humans, 30(2), 124-130. http://dx.doi.org/10.1109/3468.833093

Dubois, D. (2006). Possibility theory and statistical reasoning. Computational Statistics and Data Analysis Journal, 51(1), 47-69. http://dx.doi.org/10.1016/j.csda.2006.04.015

Dubois, D., \& Prade, H. (2011). Possibility theory and its application: Where do we stand? Mathware and Soft Computing, 18(1), 18-31.

Dubois, D., \& Prade, H. (1992). When upper probabilities are possibility measures. Fuzzy Sets and Systems Journal, 49, 65-74. http://dx.doi.org/10.1016/0165-0114(92)90110-P

Dubois, D., Prade, H., \& Smets, P. (1996). Representing partial ignorance. IEEE Trans. on Systems, Man and Cybernetics, 26(3), 361-377. http://dx.doi.org/10.1.1.39.9788

Fu, G., Butler, D., Khu, S-T., \& Sun, S. (2011). Imprecise probabilistic evaluation of sewer flooding in urban drainage systems using random set theory. Water Resources Research, 47(W0253). http://dx.doi.org/10.1029/2009WR008944

Gabbay, D. M., \& Smets, Ph. (1998). Handbook of defeasible reasoning and uncertainty management systems, Quantified representation of uncertainty and imprecision. Kluwer Academic Publishers in Netherland, 1.

Gerard, R., Kaci, S., \& Prade, H. (2007). Ranking Alternatives on the Basis of generic constraints and examples-A possibility approach. Proceedings of the $20^{\text {th }}$ International Joint Conference on Artificial Intelligence (IJCAI 2007), Hyderabad, India, 393-398. http://dx.doi.org/10.1.1.73.8099

Giles, R. (1982). Foundations of Possibility theory. Decision Processes and Fuzzy Information Journal, Northern Holland, 183-195.

Hoffman, F. O., \& Hammonds, J. S. (1994). Propagation of Uncertainty in Risk Assessments: The Need to Distinguish Between Uncertainty Due to Lack of Knowledge and Uncertainty Due to Variability. Risk Analysis, 14(5), 707-712.

Kejiang, Z. (2010). Modeling uncertainty and variability in health risk assessment of contaminated sites. DAI-B 70/07, NR49649.

Kim, J. J. (2009). Uncertainty quantification in serviceability of reinforced concrete structures. The University of New Mexico. DAI-B 70/06.

Klir, G. J., \& Folger, T. A. (1992). Fuzzy sets, Uncertainty, and Information. Prentice-Hall International Publication, Singapore.

Klir, G. J., \& Yuan, B. (1995). Fuzzy Sets and Fuzzy Logic: Theory and Applications. Prentice Hall, Upper Saddle River, New Jersey.

Kruse, R., \& Gebhardt, J. (1993). The context model-an integrating view of vagueness and uncertainty. Journal of Approximate Reasoning, 9, 283-314. http://dx.doi.org/10.1016/0888-613X(93)90014-5

Li, F.-C., \& An, L.-N. (2009). Rough set model based on possibility measure. Proceeding of the 8th International Conference on Machine Learning and Cybernetics, 2657-2662. http://dx.doi.org/10.1109/ICMLC.2009.5212645

Liu, C., \& Zhong, N. (2001). Rough problem settings for ILP dealing with imperfect data. Computational Intelligence, 17(3), 446-459. http://dx.doi.org/10.1111/0824-7935.00157

Maurice, B., Frischknecht, R., Coelho-Schwirtz, V., \& Hungerbuhler, K. (2000). Uncertainty analysis in life cycle inventory. Application to the production of electricity with French coal power plants. Cleaner Production Journal, 8(2), 95-108. http://dx.doi.org/10.1016/S0959-6526(99)00324-8

Mohamed, S., \& McCowan, A. K. (2011). Modeling project investment decisions under uncertainty using possibility theory. International Journal of Project Management, 19(4), 231-241. Elsevier Science Ltd. http://dx.doi.org/10.1016/S0263-7863(99)00077-0

Mukerji, S. (2000). A survey of some applications of the ideal of ambiguity aversion in economics. International Journal of Approximate Reasoning, 24(2-3), 221-234. http://dx.doi.org/10.1016/S0888-613X(00)00036-0

Nikolaidis, E., Chen, S., Cudney, H., Haftka, R. T., \& Rosca, R. (2004). Comparison of probability and possibility 
theory for design against catastrophic failure under uncertainty. Journal of Mechanical Design, 126(3), 386394. http://dx.doi.org/10.1115/1.1701878

Prade, H. \& Dubois, D. (1988). Representation and combination of uncertainty with belief functions and possibility measures. Journal of Computational Intelligence, $244-264$. http://dx.doi.org/10.1111/j.1467-8640.1988.tb00279

Raymond, R. T., Alvin, B. C., \& Michael, R. I. P. (2002). Application of possibility theory in life cycle inventory assessment of biofuels. International Journal of Energy Research, 26(8), 737-745. http://dx.doi.org/10.1002/er.812

Rebille, Y. (2006). Decision making over necessity measures through the Choquet integral criterion. Fuzzy Sets and Systems Journal, 157(23), 3025-3039. http://dx.doi.org/10.1016/j.fss.2006.06.001

Smets, Ph., \& Kennes, R. (1994). The transferable belief model. Artificial Intelligence Journal, 66, $191-234$. http://dx.doi.org/10.1016/0004-3702(94)90026-4

Umano, M., \& Fukami, S. (1994). Fuzzy relational algebra for possibility-distribution-fuzzy-relational model of fuzzy data. Journal of Intelligent Information System, 3(1), 7-27. http://dx.doi.org/10.1007/BF01014018

Vejnarova, J. (2002). Markov properties and factorization of possibility distribution. Annuals of Mathematics and Artificial Intelligence, 35(1-4), 357-377.

Walley, P. (1996). Measures of uncertainty in expert systems. Artificial Intelligence, 83(1), 1-58. http://dx.doi.org/10.1016/0004-3702(95)00009-7

Winkler, R. L. (1972). An Introduction to Bayesian Inference and Decision. New York: Holt, Reinhart \& Winston.

Zadeh, A. L. (1978). Fuzzy sets as a basis for theory of possibility. Fuzzy Sets and Systems Journal, 1(1), 3-28. http://dx.doi.org/10.1016/S0165-0114(99)80004-9

Zimmermann, H. J. (1996). Fuzzy Set Theory and its applications. Norwell, Massachusetts: Kluwer Academic Publishers.

http://www-static.shell.com/static/public/downloads/brochures/corporate_pkg/ scenarios/shell_energy_scenarios_ 2050.pdf

http://www.chevron.com/deliveringenergy/

http://www.iadb.org/intal/intalcdi/PE/2010/05499.pdf 